\title{
On the long-time stability of finite element solutions of the navier-stokes equations in a rotating frame of reference
}

\author{
Mine AKBAŞ* \\ Düzce Üniversitesi, Fen-Edebiyat Fakültesi,Matematik Bölümü,81620, Düzce \\ (ORCID: 0000-0002-4512-4432)
}

\begin{abstract}
This paper studies the long-time stability behavior of the Navier-Stokes equations (NSE) in a rotating frame of reference with atime accurate and adaptive finite element method. The proposed numerical scheme consists of two decoupled steps. In the first step, the Navier-Stokes equations are solved with the standard linearized backwardEuler finite element method (BE-FEM). In the second step, the approximate velocity solution obtained in the first step is post proceeded with a 2-step, linear time filter. It is proven that the approximate velocity solution is stable with respect to $L^{2}$-norm at all times. The novelty of the stability analysis is that the stability bound obtained for the approximate velocity solution does not use any Gronwall-type estimate and is polynomially dependent on the Reynolds number, which is not common in long-time stability notion. The paper also provides two numerical experiments to test the algorithm. The first numerical experiment compares the $L^{2}$-norm of the velocity solution of the proposed algorithmusing pressure-robust and non pressure-robustFE over longer time intervals. The results reveal that the scheme gives much more accurate velocity solutions with pressure-robust methods, especially for the smaller $v$. The second experiment, on the other hand, shows that the filter step increasesthe accuracy of the proposed numerical method over long-time intervals.
\end{abstract}

Keywords:Pressure-robust FEM, long-time stability, mass conservation.

\section{Dönen bir referans sisteminde verilen navier-stokes denklemlerinin sonlu elemanlarla çözümlerinin uzun zamanlı kararlılığı üzerine}

\begin{abstract}
Öz
Bu makale, Navier-Stokes denklemlerinin uzun zamanlı kararlılık davranışını zamana göre doğru ve uyarlanabilir sonlu elemanlar yöntemiile dönen bir referans sistemin içindeçalışır. Önerilen sayısal şema iki ayrıştırılmışbasamaktan oluşur. Birincibasamakta, Navier-Stokes denklemleri standart,doğrusallaştırılmış,geriEuler (GE) sonlu elemanlar yöntemi (SEY) ile çözülür. İkinci basamakta, birinci basamakta elde edilen yaklaşık hız çözümü iki adımlı, doğrusal bir zaman filtresiyle düzeltilir. Yaklaşık hız çözümünün $L^{2}$-normuna göre tüm zamanlarda kararlı olduğu ispatlanır. Kararlılık analizinin yeniliği, yaklaşık hız çözümü için elde edilen karalılık sınırının herhangi bir Gronwall tipi değerlendirmekullanmamasıve Reynolds sayısına polinomsal olarak bağımlı olmasıdır ki bu uzun zamanlı kararlılık konusunda çok yaygın değildir. Makale ayrıca algoritmayı test etmek için iki sayısal deney sunar. Birincisayısal deneynormunu daha uzun zaman aralıklarınde basınca dayanıklı ve basınca dayanıklı olmayan SE kullanarakönerilen algoritmanın hız çözümünün $L^{2}$-normunukarşılaştırır. Sonuçlar, özellikle daha küçük $v$ değerleri için, şemanınbasınca dayanıklıyöntemlerle çok daha doğru hız çözümleri verdiğini gösterir. Diğer taraftan ikinci deney ise filter basamağının uzun zaman aralıkları üzerindeönerilensayısal yöntemin doğruluğunu artırdığını gösterir.
\end{abstract}

Anahtar kelimeler: Basınca-dayanıklı SEY, uzun zamanlı kararlılık, kütlenin korunumu.

*Sorumlu yazar:mineakbas@duzce.edu.tr

Geliş Tarihi: 05.08.2019, Kabul Tarihi: 20.03.2020 


\section{Introduction}

In this paper, we focus on time dependent incompressible Navier-Stokes equations (NSE) in a rotating frame of reference which read as: for a given force fieldf, and velocity solution at $t=0, \mathbf{u}(., 0)$, find the velocity $\mathbf{u}$ and the pressure $p$ such that it holds

$$
\begin{aligned}
\mathbf{u}_{t}-v \Delta \mathbf{u}+(\mathbf{u} \cdot \nabla) \mathbf{u}+2 \Omega \times \mathbf{u}+\nabla \mathrm{p} & =\mathbf{f}+\Omega \times(\Omega \times \mathrm{r}), & & \text { in } \mathbb{D}, \\
\nabla \cdot \mathbf{u} & =0, & & \text { in } \mathbb{D}, \\
\mathbf{u}(\cdot, 0) & =\mathbf{u}^{0}, & & \text { in } \mathbb{D}, \\
\mathbf{u} & =0, & & \text { along } \partial \mathbb{D},
\end{aligned}
$$

where $\mathbb{D}$ is a bounded domain in $\mathbb{R}^{2}$ or $\mathbb{R}^{3}$ with Lipschitz continuous boundary. The first equation is the conservation of the momentum, and the second the conservation of the mass. Here $v:=1 / R e$ is the dimensionless kinematic viscosity, where $R e$ denotes the dimensionless Reynolds number, $\Omega$ is the angular velocity, $2 \Omega \times \mathbf{u}$ is the Coriolis force, $\Omega \times(\Omega \times \mathrm{r})$ is the centrifugal force where $\mathrm{r}$ denotes the distance to the orijin.

The NSE in a rotating reference frame contains two additional forces: the Coriolis and the centrifugal forces. While the Coriolis force depends on the fluid velocity, the centrifugal force is independent of the fluid unknowns; the pressure and the velocity. On the other hand, the centrifugal force is a gradient of some scalar function $\phi$, i.e., $\Omega \times(\Omega \times \mathrm{r}):=\nabla \phi$. Hence, this force can be treated as a bodyforce or included in the pressure in (1.1), which is also gradient.

There are two main difficulties in the finite element simulations of (1.1). The first one is that the small values of $v$ leads to spurious oscillations in finite element velocity solutions. This is due to the fact the continuous pressure acts as a Lagrange multiplier for the incompressibility condition, i.e., for the conservation of mass. The second one is that the discrete velocity and the pressure spaces have to satisfy some compatibility condition to guarantee the uniqueness of the finite element solutions, which is called the discrete inf-sup or the Ladyzhenskaya-Babuska-Brezzi (LBB) stability condition [5]. While the LBB condition leads to well-posed methods thatcan yield non-pressure robust discretization due to the enforcement of the divergence constraint discretely.

On the other hand, most classical FEM for the incompressible flows gives solutions which are stable only for short times. In longer time simulations, these solutions may not reflect the correct physical behavior of the flows. This is due to their linear/exponential increment with time. Therefore, designing a numerical algorithm possessing stability and accuracy at all times is a big challenge in the field of finite element theory, and the long time stability analysis.

The topic of long-time stability of the incompressible flows has attracted great interest over the years, due to the importance of predicting weather and climate events. For some valuable long-time stability results for the NSE, we cite the works of F. Tone, X. Wang, S. Gottlieb, D. Wirosoetisno and coworkers [2, 6, 15, 17, 18], and for Magnetohydrodynamics (MHD) with backward Euler schemes, and for the Boussinesq equations, the works $[4,16]$. In particular, for second order type algorithms, we give references $[3,7]$. All long-time stability results in these papers use a variant of the discrete Gronwall lemma, which produces a bound relying on the Reynolds number of the exponential form. Even though these bounds are independent of time, they are impractical for simulations of the incompressible flows with higher Reynolds' number, see [10].

Recently, a numerical approach to increase the accuracy of the BE method was introduced for ordinary differential equations in [8], and extended to the incompressible Navier-Stokes equations (NSE) in [4]. In this paper, we study the long-time stability behavior of the incompressible NSE in a rotational frame by applying this idea. The proposed method combines the standard linearly implicit BE time-stepping scheme and a linear time filter step for (3.1). In other words, the algorithm consists of two decoupled steps, and can be plugging into an existence BE-FEM solver with an only one additional line of code. 
We prove that discrete velocity solutions are long-time stable in $L_{2}$-norm providedf $\in$ $L^{\infty}\left(\mathbb{R}^{+} ; \boldsymbol{H}^{\mathbf{1}}(D)\right)$. Our stability bound does not use any Gronwall type inequality, and the dependence on the Reynolds number is polynomial, not exponential.

In view of numerical experiments, the contribution of our paper is twofold. First, the paper reveals that the pressure-robust discretization plays a crucial role on the long-time behavior of the proposed scheme's solution. Since the velocity errors in such a discretization do not depend on the continuous pressure, the velocity solutions behave in a robust way when the critical parameter $v$ attains small values; see $[8,11,12,13]$. The first numerical experimentverifies this theoretical finding for our method. The stability properties of the proposednumerical scheme is compared with the linearly implicit BE-FEM over longer time intervals in the second experiment, and the results shows that the proposed algorithm has better mass conservation, particularly for smaller viscosity values.

The rest of the paper is organized as follows. All necessary mathematical preliminaries are collected in Section 2. Section 3 presents an adaptive time filter FEM for approximating (1.1), and studies its long-time stability properties. Section 4 tests the proposed method with two numerical experiments. Section 5 gives the results of the study.

\section{Mathematical preliminaries}

In this section, we will present mathematical preliminaries and notation used throughout this paper. We consider a domain $\mathbb{D}$, and use the standard notations for Sobolev spaces and their norms as in [1]. The norm in $H^{k}(\mathbb{D})$ is labelled with $\|.\|_{k}$, and the norms in $L^{p}(\mathbb{D}), 1 \leq p<\infty$ with $\|.\|_{L} p$, and $\|$. $\|_{\infty}$ for $p=$ $\infty$. Vectors valued functions related to these spaces will be indicated with bold letters.

The natural velocity and pressure spaces for (1.1) are taken by

$$
\begin{gathered}
\mathbf{X}:=\left(H_{0}^{1}(\mathbb{D})\right)^{d}:=\left\{\mathbf{v} \in\left(L^{2}(\mathbb{D})\right)^{d}: \nabla \mathbf{v} \in\left(L^{2}(\mathbb{D})\right)^{d \times d}, \mathbf{v}=\mathbf{0} \text { on } \partial \mathbb{D}\right\}, \\
Q:=L_{0}^{2}(\mathbb{D})=\left\{q \in L^{2}(\mathbb{D}): \int_{\mathbb{D}} q d x=0\right\} .
\end{gathered}
$$

The skew-symmetrized trilinear form is labelled by

$$
\alpha(\mathbf{v}, \mathbf{s}, \mathbf{w}):=\frac{1}{2}((\mathbf{v} \cdot \nabla \mathbf{s}, \mathbf{w})-(\mathbf{v} \cdot \nabla \mathbf{w}, \mathbf{s})), \forall \mathbf{v}, \mathbf{w}, \mathbf{s} \in \mathbf{X} .
$$

We note that $\alpha(\mathbf{v}, \mathbf{s}, \mathbf{s})=0$, and $\alpha(\mathbf{v}, \mathbf{s}, \mathbf{w})$ enjoys the following property and the results [9].

Lemma 2.1. For all $\mathbf{v}, \mathbf{s}, \quad \mathbf{w} \in \mathbf{X}, \alpha(\mathbf{v}, \mathbf{s}, \mathbf{w})$ satisfies

$$
\begin{gathered}
\alpha(\mathbf{v}, \mathbf{s}, \mathbf{w})=(\mathbf{v} \cdot \nabla \mathbf{s}, \quad \mathbf{w})+\frac{1}{2}((\nabla \mathbf{v}) \mathbf{s}, \quad \mathbf{w}), \\
\alpha(\mathbf{v}, \mathbf{s}, \quad \mathbf{w}) \leq C\|\nabla \mathbf{v}\|_{L^{2}}\|\nabla \mathbf{s}\|_{L^{2}}\|\nabla \mathbf{w}\|_{L^{2}}, \\
\alpha(\mathbf{v}, \mathbf{s}, \quad \mathbf{w}) \leq C\|\mathbf{v}\|_{L^{2}}^{1 / 2}\|\nabla \mathbf{v}\|_{L^{2}}^{1 / 2}\|\nabla \mathbf{s}\|_{L^{2}}\|\nabla \mathbf{w}\|_{L^{2}},
\end{gathered}
$$

where $C$ is constant.

Forthe finite element setting, let $S_{h}$ stand for a decomposition of the domain $\mathbb{D}$ into triangles in $2 d$ or tetrahedron in $3 d$. To approximate velocity-pressure solutions of the NSE in a rotational frame, we study with conforming, inf-sup stable (LBB) finite element spaces, and label them with $\left(\mathbf{X}_{h}, Q_{h}\right) \subset(\mathbf{X}, Q)$. Recall that LBBcondition is given as follows

$$
\inf _{q_{h} \in Q_{h} \mathbf{v}_{\boldsymbol{h}} \in \mathbf{X}_{\boldsymbol{h}}} \frac{\left(q_{h}, \boldsymbol{\nabla} \cdot \mathbf{v}_{\boldsymbol{h}}\right)}{\left\|q_{h}\right\|_{L^{2}}\left\|\boldsymbol{\nabla} \mathbf{v}_{\boldsymbol{h}}\right\|_{L^{2}}} \geq \boldsymbol{\beta}>\mathbf{0},
$$


where $\beta$ is independent on $h$. The discretely divergence- free subspace is given

$$
\mathbf{V}_{h}:=\left\{\mathbf{v}_{\boldsymbol{h}} \in \mathbf{X}_{\boldsymbol{h}}:\left(q_{h}, \boldsymbol{\nabla} \cdot \mathbf{v}_{h}\right)=0, \forall q_{h} \in Q_{\boldsymbol{h}}\right\}
$$

The dual space of $\mathbf{V}_{h}$ will be identified by $\mathbf{V}_{h}^{*}$, and is endowed with the following dual form:

$$
\|\mathbf{w}\|_{\mathbf{v}_{h}^{*}}:=\sup _{\mathrm{v}_{\boldsymbol{h}} \in \mathbf{v}_{\boldsymbol{h}}} \frac{\left|\left(\mathbf{w}, \mathbf{v}_{h}\right)\right|}{\left\|\nabla_{\mathrm{v}_{\boldsymbol{h}}}\right\|_{L^{2}}} .
$$

For a chosen time step $\Delta t$, we introduce the notation $t^{n+1}:=(n+1) \Delta t$, and indicate $\mathbf{v}^{n+1}:=$ $\mathbf{v}\left(t^{n+1}\right)$. Our stability analysis frequently uses the Young's inequality, which is given below [9].

Lemma 2.2(Young's Inequality) Let $\lambda, \alpha$ be non-negative real numbers. Then for any $\varepsilon>0$

$$
\lambda \alpha \leq \frac{\varepsilon}{p} \lambda^{p}+\frac{\varepsilon^{-\frac{q}{p}}}{q} \alpha^{q}
$$

where $\frac{1}{p}+\frac{1}{q}=1$ with $p, q \in[1, \infty)$.

\section{Numerical scheme}

Algorithm 3.1. Let body force $\mathbf{f}$, initial velocities $\mathbf{u}^{0}, \mathbf{u}^{1}$ be given. Set $\mathbf{u}_{h}^{0}, \mathbf{u}_{h}^{1}$ to be the nodalinterpolant of $\mathbf{u}^{0}, \mathbf{u}^{1} \in \mathbf{X}_{\boldsymbol{h}}$, and selecta time step $\Delta t>0$. Then for any $n \geq 0$, find $\left(\mathbf{u}_{h}^{n+1}, p_{h}^{n+1}\right) \in\left(\mathbf{X}_{h}, Q_{h}\right)$ via the following two steps:

Step 1: Compute $\left(\widehat{\mathbf{u}}_{h}^{n+1}, p_{h}^{n+1}\right) \in\left(\mathbf{X}_{h}, Q_{h}\right)$ such that for each $\left(\mathbf{v}_{\boldsymbol{h}}, q_{h}\right) \in\left(\mathbf{X}_{h}, Q_{h}\right)$ it holds

$$
\begin{aligned}
& \left(\frac{\widehat{\mathbf{u}}_{h}^{n+1}-\mathbf{u}_{h}^{n}}{\Delta t}, \mathbf{v}_{\boldsymbol{h}}\right)+v\left(\nabla \widehat{\mathbf{u}}_{h}^{n+1}, \nabla \mathbf{v}_{\boldsymbol{h}}\right)+b\left(2 \mathbf{u}_{h}^{n}-\mathbf{u}_{h}^{n-1} \cdot \widehat{\mathbf{u}}_{h}^{n+1}, \mathbf{v}_{\boldsymbol{h}}\right)-\left(p_{h}^{n+1}, \nabla \cdot \mathbf{v}_{\boldsymbol{h}}\right) \\
& +\left(2 \Omega \times \widehat{\mathbf{u}}_{h}^{n+1}, \mathbf{v}_{\boldsymbol{h}}\right)=\left(\mathbf{f}^{n+1}, \mathbf{v}_{\boldsymbol{h}}\right), \\
& \left(\nabla \cdot \widehat{\mathbf{u}}_{h}^{n+1}, q_{h}\right)=0 .
\end{aligned}
$$

Step 2: Compute $\mathbf{u}_{h}^{n+1} \in \mathbf{X}_{\boldsymbol{h}}$ satisfyingfor each $\boldsymbol{\chi}_{h} \in \mathbf{X}_{h}$,

$$
\left(\mathbf{u}_{h}^{n+1}, \chi_{h}\right)=\left(\widehat{\mathbf{u}}_{h}^{n+1}, \chi_{h}\right)-\frac{1}{3}\left(\widehat{\mathbf{u}}_{h}^{n+1}-2 \mathbf{u}_{h}^{n}+\mathbf{u}_{h}^{n-1}, \chi_{h}\right)
$$

Assume that the time step is constant. For any $\mathbf{v}_{\boldsymbol{h}} \in \mathbf{X}_{h}$, insertthe following in the first step of Algorithm 3.1

$$
\left(\widehat{\mathbf{u}}_{h}^{n+1}, \mathbf{v}_{\boldsymbol{h}}\right)=\frac{1}{2}\left(3 \mathbf{u}_{h}^{n+1}-2 \mathbf{u}_{h}^{n}+\mathbf{u}_{h}^{n-1}, \mathbf{v}_{\boldsymbol{h}}\right)
$$

Then we have the following equivalence method.

Algorithm 3.2. Let body forcef, initial velocities $\mathbf{u}^{0}, \mathbf{u}^{1}$ be given. Set $\mathbf{u}_{h}^{0}, \mathbf{u}_{h}^{1}$ to be the nodalinterpolant of $\mathbf{u}^{0}, \mathbf{u}^{1} \in\left(\mathbf{X}_{h}, Q_{h}\right)$ and select a time step $\Delta t>0$. Then for any $n \geq 0$, find $\left(\mathbf{u}_{h}^{n+1}, p_{h}^{n+1}\right) \in$ $\left(\mathbf{X}_{h}, Q_{h}\right)$ such that for al $l\left(\mathbf{v}_{\boldsymbol{h}}, q_{\boldsymbol{h}}\right) \in\left(\mathbf{X}_{h}, Q_{h}\right)$ it holds 


$$
\begin{aligned}
& \left(\frac{\delta\left[\mathbf{u}_{h}^{n+1}\right]}{\Delta t}, \mathbf{v}_{h}\right)+v\left(\nabla E\left[\mathbf{u}_{h}^{n+1}\right], \mathbf{v}_{h}\right)+b\left(2 \mathbf{u}_{h}^{n}-\mathbf{u}_{h}^{n-1}, E\left[\mathbf{u}_{h}^{n+1}\right], \mathbf{v}_{h}\right)-\left(p_{h}^{n+1}, \boldsymbol{\nabla} \cdot \mathbf{v}_{h}\right) \\
& +\left(2 \Omega \times E\left[\mathbf{u}_{h}^{n+1}\right], \mathbf{v}_{h}\right)=\left(\mathbf{f}^{n+1}, \mathbf{v}_{h}\right), \\
& \left(\nabla \cdot E\left[\mathbf{u}_{h}^{n+1}\right], q_{h}\right)=0 .
\end{aligned}
$$

where

$$
\delta\left[\mathbf{u}_{h}^{n+1}\right]:=\frac{3}{2} \mathbf{u}_{h}^{n+1}-2 \mathbf{u}_{h}^{n}+\frac{1}{2} \mathbf{u}_{h}^{n-1}, \quad E\left[\mathbf{u}_{h}^{n+1}\right]:=\frac{3}{2} \mathbf{u}_{h}^{n+1}-\mathbf{u}_{h}^{n}+\frac{1}{2} \mathbf{u}_{h}^{n-1} .
$$

Remark 3.1. Notice that Algorithm 3.2 is not the standard linearizedtwo-step backward difference formula (BDF2)since it onlyusesBDF2 for the time derivative term, and does not use that for the remaining terms.

We now prove that velocity solution of Algorithm 3.2 is long-time stable.

Lemma 3.1. Letf $\in \mathbf{L}^{\infty}\left(\mathbb{R}^{+} ; \mathbf{V}_{\mathbf{h}}^{*}\right), \mathbf{u}^{0}, \mathbf{u}^{1} \in \boldsymbol{H}^{\mathbf{1}}(\mathbb{D})$, and $\Delta t>0$ be any time step satisfying $\Delta t \leq \frac{5 h^{2}}{v c_{1}^{2}}$. Then for any non-negative integer $n$, the velocity solution of Algorithm 3.2 satisfies the bound

$$
\begin{gathered}
\left\|\mathbf{u}_{h}^{n+1}\right\|_{L^{2}}^{2}+\left\|2 \mathbf{u}_{h}^{n+1}-\mathbf{u}_{h}^{n}\right\|^{2}+\left\|\mathbf{u}_{h}^{n+1}-\mathbf{u}_{h}^{n}\right\|^{2}+\frac{v \Delta t}{4}\left\|\mathbf{u}_{h}^{n+1}\right\|^{2}+\frac{v \Delta t}{2} \sum_{k=0}^{n}\left(\frac{1}{1+\gamma}\right)^{n-k}\left\|\nabla \mathbf{u}_{h}^{k+1}\right\|^{2} \\
\leq(1+\gamma)^{-(n+1)}\left(\left\|\mathbf{u}_{h}^{1}\right\|^{2}+\left\|2 \mathbf{u}_{h}^{1}-\mathbf{u}_{h}^{0}\right\|^{2}+\left\|\mathbf{u}_{h}^{1}-\mathbf{u}_{h}^{0}\right\|^{2}+\frac{v \Delta t}{4}\left\|\mathbf{u}_{h}^{1}\right\|^{2}\right) \\
+\max \left\{2 \Delta t, \frac{88 C_{P}^{2}}{v}\right\} v^{-1}\|\mathbf{f}\|_{\mathbf{L}^{\infty}\left(\mathbb{R}^{+} ; \mathbf{v}_{\mathbf{h}}^{*}\right)}^{2}
\end{gathered}
$$

wherer $=\min \left\{\frac{1}{2}, \frac{v \Delta t}{44 C_{P}^{2}}\right\}$.

Proof. To prove the bound on the velocity, first set $\left(\mathbf{v}_{\boldsymbol{h}}, q_{h}\right)=\left(E\left[\mathbf{u}_{h}^{n+1}\right], p_{h}^{n+1}\right)$ in (3.3)-(3.4) which vanishes the non linear term, the Coriolis force, and the pressure term

$$
b\left(2 \mathbf{u}_{h}^{n}-\mathbf{u}_{h}^{n-1}, E\left[\mathbf{u}_{h}^{n+1}\right], \quad E\left[\mathbf{u}_{h}^{n+1}\right]\right),\left(2 \Omega \times E\left[\mathbf{u}_{h}^{n+1}\right], E\left[\mathbf{u}_{h}^{n+1}\right], \quad\left(p_{h}^{n+1}, \quad \nabla . E\left[\mathbf{u}_{h}^{n+1}\right]\right)\right) .
$$

Then use the algebraic identity

$$
\begin{aligned}
\left(\frac{3 a-4 b+c}{2}\right) & \left(\frac{3 a-2 b+c}{2}\right) \\
& =\frac{1}{4}\left[a^{2}+(2 a-b)^{2}+(a-b)^{2}\right]-\frac{1}{4}\left[b^{2}+(2 b-c)^{2}+(b-c)^{2}\right] \\
& +\frac{3}{4}(a-2 b+c)^{2}
\end{aligned}
$$

to get

$$
\begin{aligned}
\frac{1}{4 \Delta t}\left(\left\|\mathbf{u}_{h}^{n+1}\right\|^{2}\right. & \left.-\left\|\mathbf{u}_{h}^{n}\right\|^{2}+\left\|2 \mathbf{u}_{h}^{n+1}-\mathbf{u}_{h}^{n}\right\|^{2}+\left\|2 \mathbf{u}_{h}^{n}-\mathbf{u}_{h}^{n-1}\right\|^{2}+\left\|\mathbf{u}_{h}^{n+1}-\mathbf{u}_{h}^{n}\right\|^{2}-\left\|\mathbf{u}_{h}^{n}-\mathbf{u}_{h}^{n-1}\right\|^{2}\right) \\
& +\frac{3}{4 \Delta t}\left\|\mathbf{u}_{h}^{n+1}-2 \mathbf{u}_{h}^{n}+\mathbf{u}_{h}^{n-1}\right\|^{2}+v\left\|\nabla E\left[\mathbf{u}_{h}^{n+1}\right]\right\|^{2} \leq\left|\left(\mathbf{f}^{n+1}, E\left[\mathbf{u}_{h}^{n+1}\right]\right)\right|
\end{aligned}
$$

Applying the Cauchy-Schwarz and Young's Inequality (2.1) with $\varepsilon=v$ on the forcing term yields 


$$
\left|\left(\mathbf{f}^{n+1}, E\left[\mathbf{u}_{h}^{n+1}\right]\right)\right| \leq\left\|\mathbf{f}^{n+1}\right\|_{\mathbf{v}_{h}^{*}}\left\|\nabla E\left[\mathbf{u}_{h}^{n+1}\right]\right\|_{L^{2}} \leq \frac{v^{-1}}{2}\|\mathbf{f}\|_{\mathbf{L}^{\infty}\left(\mathbb{R}^{+} ; \mathbf{v}_{\mathbf{h}}^{*}\right)}^{2}+\frac{v}{2}\left\|\nabla E\left[\mathbf{u}_{h}^{n+1}\right]\right\|^{2}
$$

Inserting this estimate into (3.5), reducing and multiplying by $4 \Delta t$ produces

$$
\begin{aligned}
\left\|\mathbf{u}_{h}^{n+1}\right\|^{2} & +\left\|2 \mathbf{u}_{h}^{n+1}-\mathbf{u}_{h}^{n}\right\|^{2}+\left\|\mathbf{u}_{h}^{n+1}-\mathbf{u}_{h}^{n}\right\|^{2}+3\left\|\mathbf{u}_{h}^{n+1}-2 \mathbf{u}_{h}^{n}+\mathbf{u}_{h}^{n-1}\right\|^{2}+2 v \Delta t\left\|\nabla E\left[\mathbf{u}_{h}^{n+1}\right]\right\|^{2} \\
& \leq\left\|\mathbf{u}_{h}^{n}\right\|^{2}+\left\|2 \mathbf{u}_{h}^{n}-\mathbf{u}_{h}^{n-1}\right\|^{2}+\left\|\mathbf{u}_{h}^{n}-\mathbf{u}_{h}^{n-1}\right\|^{2}+2 v^{-1} \Delta t\|\mathbf{f}\|_{\mathbf{L}^{\infty}\left(\mathbb{R}^{+} ; \mathbf{v}_{\mathbf{h}}^{*}\right)}
\end{aligned}
$$

Now rewrite the last left hand side term as follows

$$
\begin{aligned}
& 2 v \Delta t\left\|\nabla E\left[\mathbf{u}_{h}^{n+1}\right]\right\|^{2}=2 v \Delta t\left(\boldsymbol{\nabla}\left(\frac{3 \mathbf{u}_{h}^{n+1}-2 \mathbf{u}_{h}^{n}+\mathbf{u}_{h}^{n-1}}{2}\right), \boldsymbol{\nabla}\left(\frac{3 \mathbf{u}_{h}^{n+1}-2 \mathbf{u}_{h}^{n}+\mathbf{u}_{h}^{n-1}}{2}\right)\right) \\
& =2 v \Delta t\left(\boldsymbol{\nabla} \mathbf{u}_{h}^{n+1}+\boldsymbol{\nabla}\left(\frac{\mathbf{u}_{h}^{n+1}-2 \mathbf{u}_{h}^{n}+\mathbf{u}_{h}^{n-1}}{2}\right), \nabla \mathbf{u}_{h}^{n+1}+\boldsymbol{\nabla}\left(\frac{\mathbf{u}_{h}^{n+1}-2 \mathbf{u}_{h}^{n}+\mathbf{u}_{h}^{n-1}}{2}\right)\right) \\
& =2 v \Delta t\left\|\nabla \mathbf{u}_{h}^{n+1}\right\|^{2}+\frac{v \Delta t}{2}\left\|\nabla\left(\mathbf{u}_{h}^{n+1}-2 \mathbf{u}_{h}^{n}+\mathbf{u}_{h}^{n-1}\right)\right\|^{2}+v \Delta t\left(\boldsymbol{\nabla} \mathbf{u}_{h}^{n+1}, \boldsymbol{\nabla}\left(\mathbf{u}_{h}^{n+1}-2 \mathbf{u}_{h}^{n}+\mathbf{u}_{h}^{n-1}\right)\right) .
\end{aligned}
$$

Now use this estimate in (3.6) to get

$$
\begin{aligned}
\left\|\mathbf{u}_{h}^{n+1}\right\|^{2}+ & \left\|2 \mathbf{u}_{h}^{n+1}-\mathbf{u}_{h}^{n}\right\|^{2}+\left\|\mathbf{u}_{h}^{n+1}-\mathbf{u}_{h}^{n}\right\|^{2}+3\left\|\mathbf{u}_{h}^{n+1}-2 \mathbf{u}_{h}^{n}+\mathbf{u}_{h}^{n-1}\right\|^{2}+2 v \Delta t\left\|\nabla \mathbf{u}_{h}^{n+1}\right\|^{2} \\
& +\frac{v \Delta t}{2}\left\|\boldsymbol{\nabla}\left(\mathbf{u}_{h}^{n+1}-2 \mathbf{u}_{h}^{n}+\mathbf{u}_{h}^{n-1}\right)\right\|^{2} \\
\leq & \left\|\mathbf{u}_{h}^{n}\right\|^{2}+\left\|2 \mathbf{u}_{h}^{n}-\mathbf{u}_{h}^{n-1}\right\|^{2}+\left\|\mathbf{u}_{h}^{n}-\mathbf{u}_{h}^{n-1}\right\|^{2}+2 v^{-1} \Delta t\|\mathbf{f}\|_{\mathbf{L}^{\infty}\left(\mathbb{R}^{+} ; \mathbf{v}_{\mathbf{h}}^{*}\right)}^{(3.7)} \\
& +v \Delta t\left|\left(\nabla \mathbf{u}_{h}^{n+1}, \quad \boldsymbol{\nabla}\left(\mathbf{u}_{h}^{n+1}-2 \mathbf{u}_{h}^{n}+\mathbf{u}_{h}^{n-1}\right)\right)\right|
\end{aligned}
$$

Now apply the Cauchy-Schwarz and Young's inequalities on the last term to obtain

$$
\begin{aligned}
& v \Delta t\left|\left(\boldsymbol{\nabla} \mathbf{u}_{h}^{n+1}, \boldsymbol{\nabla}\left(\mathbf{u}_{h}^{n+1}-2 \mathbf{u}_{h}^{n}+\mathbf{u}_{h}^{n-1}\right)\right)\right| \leq v \Delta t\left\|\nabla \mathbf{u}_{h}^{n+1}\right\| C_{I} h^{-1}\left\|\mathbf{u}_{h}^{n+1}-2 \mathbf{u}_{h}^{n}+\mathbf{u}_{h}^{n-1}\right\| \\
& \leq \frac{v \Delta t}{2}\left\|\nabla \mathbf{u}_{h}^{n+1}\right\|^{2}+\frac{v \Delta t C_{I}^{2} h^{-2}}{2}\left\|\mathbf{u}_{h}^{n+1}-2 \mathbf{u}_{h}^{n}+\mathbf{u}_{h}^{n-1}\right\|^{2}
\end{aligned}
$$

Plug this estimate into (3.7) to get

$$
\begin{aligned}
\left\|\mathbf{u}_{h}^{n+1}\right\|^{2}+\left\|2 \mathbf{u}_{h}^{n+1}-\mathbf{u}_{h}^{n}\right\|^{2}+\left\|\mathbf{u}_{h}^{n+1}-\mathbf{u}_{h}^{n}\right\|^{2}+\left(3-\frac{v \Delta t C_{I}^{2} h^{-2}}{2}\right)\left\|\mathbf{u}_{h}^{n+1}-2 \mathbf{u}_{h}^{n}+\mathbf{u}_{h}^{n-1}\right\|^{2} \\
+\frac{3 v \Delta t}{2}\left\|\nabla \mathbf{u}_{h}^{n+1}\right\|^{2}+\frac{v \Delta t}{2}\left\|\nabla\left(\mathbf{u}_{h}^{n+1}-2 \mathbf{u}_{h}^{n}+\mathbf{u}_{h}^{n-1}\right)\right\|^{2} \\
\leq\left\|\mathbf{u}_{h}^{n}\right\|^{2}+\left\|2 \mathbf{u}_{h}^{n}-\mathbf{u}_{h}^{n-1}\right\|^{2}+\left\|\mathbf{u}_{h}^{n}-\mathbf{u}_{h}^{n-1}\right\|^{2}+2 v^{-1} \Delta t\|\mathbf{f}\|_{\mathbf{L}^{\infty}\left(\mathbb{R}^{+} ; \mathbf{v}_{\mathbf{h}}^{*}\right)} \\
+v \Delta t\left|\left(\nabla \mathbf{u}_{h}^{n+1}, \boldsymbol{\nabla}\left(\mathbf{u}_{h}^{n+1}-2 \mathbf{u}_{h}^{n}+\mathbf{u}_{h}^{n-1}\right)\right)\right| .
\end{aligned}
$$

Now using the assumption on the time step, dropping the non-negative fourth and sixth left hand side terms, and adding $\frac{v \Delta t}{4}\left\|\nabla \mathbf{u}_{h}^{n}\right\|^{2}$ to the both side yields 


$$
\begin{aligned}
&\left\|\mathbf{u}_{h}^{n+1}\right\|^{2}+\left\|2 \mathbf{u}_{h}^{n+1}-\mathbf{u}_{h}^{n}\right\|^{2}+\left\|\mathbf{u}_{h}^{n+1}-\mathbf{u}_{h}^{n}\right\|^{2}+\frac{v \Delta t}{4}\left\|\nabla \mathbf{u}_{h}^{n+1}\right\|^{2}+\frac{v \Delta t}{2}\left\|\nabla \mathbf{u}_{h}^{n+1}\right\|^{2} \\
&+\left[\frac{v \Delta t}{4}\left(\left\|\nabla \mathbf{u}_{h}^{n+1}\right\|^{2}+\left\|\nabla \mathbf{u}_{h}^{n}\right\|^{2}\right)+\frac{v \Delta t}{2}\left\|\nabla \mathbf{u}_{h}^{n+1}\right\|^{2}\right] \\
& \leq\left\|\mathbf{u}_{h}^{n}\right\|^{2}+\left\|2 \mathbf{u}_{h}^{n}-\mathbf{u}_{h}^{n-1}\right\|^{2}+\left\|\mathbf{u}_{h}^{n}-\mathbf{u}_{h}^{n-1}\right\|^{2}+\frac{v \Delta t}{4}\left\|\nabla \mathbf{u}_{h}^{n}\right\|^{2} \\
&+2 v^{-1} \Delta t\|\mathbf{f}\|_{\mathbf{L}^{\infty}\left(\mathbb{R}^{+} ; \mathbf{v}_{\mathbf{h}}^{*}\right)}^{2}
\end{aligned}
$$

Now use the algebraic inequality $\|a+b\|^{2} \leq 2\left(\|a\|^{2}+\|b\|^{2}\right)$ to get

$$
\begin{gathered}
\left\|2 \mathbf{u}_{h}^{n+1}-\mathbf{u}_{h}^{n}\right\|^{2} \leq 2\left(\left\|2 \mathbf{u}_{h}^{n+1}\right\|^{2}+\left\|\mathbf{u}_{h}^{n}\right\|^{2}\right)=8\left\|\mathbf{u}_{h}^{n+1}\right\|^{2}+2\left\|\mathbf{u}_{h}^{n}\right\|^{2}, \\
\left\|\mathbf{u}_{h}^{n+1}-\mathbf{u}_{h}^{n}\right\|^{2} \leq 2\left(\left\|\mathbf{u}_{h}^{n+1}\right\|^{2}+\left\|\mathbf{u}_{h}^{n}\right\|^{2}\right)=2\left\|\mathbf{u}_{h}^{n+1}\right\|^{2}+2\left\|\mathbf{u}_{h}^{n}\right\|^{2},
\end{gathered}
$$

and sum these two inequalities by adding $\left\|\mathbf{u}_{h}^{n+1}\right\|^{2}$ to the both sides. This produces

$$
\left\|\mathbf{u}_{h}^{n+1}\right\|^{2}+\left\|2 \mathbf{u}_{h}^{n+1}-\mathbf{u}_{h}^{n}\right\|^{2}+\left\|\mathbf{u}_{h}^{n+1}-\mathbf{u}_{h}^{n}\right\|^{2} \leq 11\left\|\mathbf{u}_{h}^{n+1}\right\|^{2}+4\left\|\mathbf{u}_{h}^{n}\right\|^{2} \leq 11\left(\left\|\mathbf{u}_{h}^{n+1}\right\|^{2}+\left\|\mathbf{u}_{h}^{n}\right\|^{2}\right) .
$$

Using the Poincare-Friedrich inequality for the last term on the left hand side of (3.9) together with this estimate gives

$$
\begin{aligned}
\frac{v \Delta t}{4}\left(\left\|\nabla \mathbf{u}_{h}^{n+1}\right\|^{2}+\left\|\nabla \mathbf{u}_{h}^{n}\right\|^{2}\right) & +\frac{v \Delta t}{2}\left\|\nabla \mathbf{u}_{h}^{n+1}\right\|^{2} \\
& \geq \frac{v \Delta t}{4 C_{P}^{2}}\left(\left\|\mathbf{u}_{h}^{n+1}\right\|^{2}+\left\|\mathbf{u}_{h}^{n}\right\|^{2}\right)+\frac{v \Delta t}{2}\left\|\nabla \mathbf{u}_{h}^{n+1}\right\|^{2} \\
\geq & \frac{v \Delta t}{44 C_{P}^{2}}\left(\left\|\mathbf{u}_{h}^{n+1}\right\|^{2}+\left\|2 \mathbf{u}_{h}^{n+1}-\mathbf{u}_{h}^{n}\right\|^{2}+\left\|\mathbf{u}_{h}^{n+1}-\mathbf{u}_{h}^{n}\right\|^{2}\right)+\frac{v \Delta t}{2}\left\|\nabla \mathbf{u}_{h}^{n+1}\right\|^{2} \\
\geq & \min \left\{\frac{1}{2}, \frac{v \Delta t}{44 C_{P}^{2}}\right\}\left(\left\|\mathbf{u}_{h}^{n+1}\right\|^{2}+\left\|2 \mathbf{u}_{h}^{n+1}-\mathbf{u}_{h}^{n}\right\|^{2}+\left\|\mathbf{u}_{h}^{n+1}-\mathbf{u}_{h}^{n}\right\|^{2}+\frac{v \Delta t}{2}\left\|\nabla \mathbf{u}_{h}^{n+1}\right\|^{2}\right) .
\end{aligned}
$$

Now insert this estimate into (3.9), denote $\gamma:=\min \left\{\frac{1}{2}, \frac{v \Delta t}{44 C_{P}^{2}}\right\}$. This yields

$$
\begin{aligned}
(1+\gamma)\left(\left\|\mathbf{u}_{h}^{n+1}\right\|^{2}\right. & \left.+\left\|2 \mathbf{u}_{h}^{n+1}-\mathbf{u}_{h}^{n}\right\|^{2}+\left\|\mathbf{u}_{h}^{n+1}-\mathbf{u}_{h}^{n}\right\|^{2}+\frac{v \Delta t}{4}\left\|\nabla \mathbf{u}_{h}^{n+1}\right\|^{2}\right)+\frac{v \Delta t}{2}\left\|\nabla \mathbf{u}_{h}^{n+1}\right\|^{2} \\
\leq & \left(\left\|\mathbf{u}_{h}^{n}\right\|^{2}+\left\|2 \mathbf{u}_{h}^{n}-\mathbf{u}_{h}^{n-1}\right\|^{2}+\left\|\mathbf{u}_{h}^{n}-\mathbf{u}_{h}^{n-1}\right\|^{2}+\frac{v \Delta t}{4}\left\|\nabla \mathbf{u}_{h}^{n}\right\|^{2}\right) \\
& +2 v^{-1} \Delta t\|\mathbf{f}\|_{\mathbf{L}^{\infty}\left(\mathbb{R}^{+} ; \mathbf{v}_{\mathbf{h}}^{*}\right)}^{2}
\end{aligned}
$$

Fix an integer $M>0$, and divide the above inequality by $(1+\gamma)^{M-n}$ to obtain

$$
\begin{gathered}
(1+\gamma)^{1-M+n}\left(\left\|\mathbf{u}_{h}^{n+1}\right\|^{2}+\left\|2 \mathbf{u}_{h}^{n+1}-\mathbf{u}_{h}^{n}\right\|^{2}+\left\|\mathbf{u}_{h}^{n+1}-\mathbf{u}_{h}^{n}\right\|^{2}+\frac{v \Delta t}{4}\left\|\nabla \mathbf{u}_{h}^{n+1}\right\|_{L^{2}}^{2}\right) \\
+(1+\gamma)^{-M+n} \frac{v \Delta t}{2}\left\|\nabla \mathbf{u}_{h}^{n+1}\right\|_{L^{2}}^{2} \\
\leq(1+\gamma)^{-M+n}\left(\left\|2 \mathbf{u}_{h}^{n}-\mathbf{u}_{h}^{n-1}\right\|^{2}+\left\|\mathbf{u}_{h}^{n}-\mathbf{u}_{h}^{n-1}\right\|^{2}+\frac{v \Delta t}{4}\left\|\nabla \mathbf{u}_{h}^{n}\right\|_{L^{2}}^{2}\right) \\
+2(1+\gamma)^{-M+n} v^{-1} \Delta t\|\mathbf{f}\|_{\mathbf{L}^{\infty}\left(\mathbb{R}^{+} ; \mathbf{v}_{\mathbf{h}}^{*}\right)}^{2}
\end{gathered}
$$

Summing up for $n=0,1, \ldots, M-1$ and reducing yields 


$$
\begin{gathered}
\left\|\mathbf{u}_{h}^{M}\right\|^{2}+\left\|2 \mathbf{u}_{h}^{M}-\mathbf{u}_{h}^{M-1}\right\|^{2}+\left\|\mathbf{u}_{h}^{M}-\mathbf{u}_{h}^{M-1}\right\|^{2}+\frac{v \Delta t}{4}\left\|\nabla \mathbf{u}_{h}^{M}\right\|_{L^{2}}^{2}+\frac{v \Delta t}{2} \sum_{n=0}^{M-1}\left(\frac{1}{1+\gamma}\right)^{M-n}\left\|\nabla \mathbf{u}_{h}^{n+1}\right\|_{L^{2}}^{2} \\
\leq(1+\gamma)^{-M}\left(\left\|\mathbf{u}_{h}^{1}\right\|^{2}+\left\|2 \mathbf{u}_{h}^{1}-\mathbf{u}_{h}^{0}\right\|^{2}+\left\|\mathbf{u}_{h}^{1}-\mathbf{u}_{h}^{0}\right\|^{2}+\frac{v \Delta t}{4}\left\|\nabla \mathbf{u}_{h}^{1}\right\|_{L^{2}}^{2}\right) \\
+2 v^{-1} \Delta t\|\mathbf{f}\|_{\mathbf{L}^{\infty}\left(\mathbb{R}^{+} ; \mathbf{v}_{\mathbf{h}}^{*}\right)} \sum_{n=0}^{M-1}(1+\gamma)^{-M+n} .
\end{gathered}
$$

Using the fact that

$$
\begin{gathered}
2 \Delta t\left((1+\gamma)^{-1}+(1+\gamma)^{-2}+\cdots+(1+\gamma)^{-(n+1)}\right)=2 \Delta t(1+\gamma)^{-1}\left(\frac{1-(1+\gamma)^{-(n+1)}}{1-(1+\gamma)^{-1}}\right) \\
\leq 2 \Delta t(1+\gamma)^{-1}\left(\frac{1}{1-(1+\gamma)^{-1}}\right) \\
\leq \max \left\{\Delta t, \frac{44 C_{P}^{2}}{v}\right\}
\end{gathered}
$$

finishes the proof.

\section{Numerical experiments}

In this section, we present two numerical experiments. Each of them uses the same test problem. The system is studied on a unit square domain $\mathbb{D}:=(\mathbf{0}, \mathbf{1})^{\mathbf{2}}$ with a constant inflow $\mathbf{u}:=(\mathbf{0}, \mathbf{1})$, and angular velocity $\boldsymbol{\Omega} .=(0,0, y)$. The pressure is chosen as a quadratic polynomial $p(x, y)=-y^{2}+1 / 3$ such that $\int_{\mathbb{D}} p \boldsymbol{d} \boldsymbol{x} \boldsymbol{d} \boldsymbol{y}=0$. In this case, right hand side forcing term $\mathbf{f}$ would be zero.

\subsection{Mass conservation}

In this numerical experiment, we will show that divergence-free FEM behaves in a robust way, and has better mass conservation when the criticial parameter $v \rightarrow 0$. To show that, we compute solutions of Algorithm 3.1 with divergence-free, the Scott-Vogelius (SV), and non-divergence free elements, the Taylor-Hood (TH), Mini and Crouzeix-Raviart (CR), on the same 16x16 barycenter refined uniform mesh of domain $\mathbb{D}$. We fix end time to $\mathrm{T}=100$, and take time step $\mathrm{dt}=0.05$. (We also run $\mathrm{dt}=0.01, \mathrm{dt}=$ 0.1 , and got the similar results.) For each element choice, we compute $\left\|\mathbf{u}_{\boldsymbol{h}}^{n+1}\right\|_{L^{2}}$ for varying $\boldsymbol{v}$. As can be seen from Table 1, all elements give the exact velocity solution for $\boldsymbol{v}=1.0, \mathbf{1 0}^{-3}$ However, only the SV-FE solutions behaves in a robust way for smaller values of $\boldsymbol{v}$. (see also Figure 1 and Figure 2.)

We also compute the divergence of approximate velocity solutions for these element choice at end time $\mathrm{T}=100$ taking the same flow parameters. The results in Table 2 reveal that Taylor-Hood and Mini elements lead to very poor mass conservation, but CR and SV strong mass conservation, which is due to the fact that CR and SV enforce the divergence constraint strongly, not discretely.

Table 1. Discrete velocities in $\boldsymbol{L}_{2}$-norm at $\mathrm{T}=100$ for varying $\boldsymbol{v}$.

\begin{tabular}{ccccc}
\hline & $\mathrm{TH}\left(\boldsymbol{P}_{\mathbf{2}}, P_{1}\right)$ & $\operatorname{Mini}\left(\boldsymbol{P}_{1}^{\text {bub }}, P_{\mathbf{1}}\right)$ & $\mathrm{CR}\left(\boldsymbol{P}_{\mathbf{1}}^{n c}, P_{\mathbf{0}}\right)$ & $\mathrm{SV}\left(\boldsymbol{P}_{\mathbf{2}}, P_{1}^{d c}\right)$ \\
\hline $\boldsymbol{v}$ & $\left\|\mathbf{u}_{h}(T)\right\|_{L^{2}}$ & $\left\|\mathbf{u}_{h}(T)\right\|_{\mathbf{L}^{2}}$ & $\left\|\mathbf{u}_{h}(T)\right\|_{L^{2}}$ & $\left\|\mathbf{u}_{h}(T)\right\|_{L^{2}}$ \\
\hline $10^{0}$ & 1.0 & 1.0 & 1.0 & 1.0 \\
$10^{-2}$ & 1.0 & 1.0 & 1.0 & 1.0 \\
$10^{-3}$ & 1.2597 & 1.0 & 1.0795 & 1.0 \\
$10^{-4}$ & 1.7372 & 1.0980 & 1.1432 & 1.0 \\
$10^{-5}$ & 2.0841 & 1.3297 & 1.1562 & 1.0 \\
$10^{-6}$ & 2.1467 & 1.4129 & 1.1524 & 1.0 \\
\hline
\end{tabular}


Table 2.Divergence of velocity solutions in $\boldsymbol{L}_{2}$-norm at $\mathrm{T}=100$ for varying $v$.

\begin{tabular}{ccccc}
\hline & $\mathrm{TH}\left(P_{2}, P_{\mathbf{1}}\right)$ & $\operatorname{Mini}\left(\boldsymbol{P}_{1}^{b u b}, P_{\mathbf{1}}\right)$ & $\operatorname{CR}\left(\boldsymbol{P}_{\mathbf{1}}^{\text {nc }}, P_{\mathbf{0}}\right)$ & $\mathrm{SV}\left(\boldsymbol{P}_{2}, P_{1}^{d c}\right)$ \\
\hline $\boldsymbol{v}$ & $\left\|\left(\nabla \cdot \mathbf{u}_{h}\right)(T)\right\|_{L^{2}}$ & $\left\|\left(\nabla \cdot \mathbf{u}_{h}\right)(T)\right\|$ & $\left\|\left(\nabla \cdot \mathbf{u}_{h}\right)(T)\right\|$ & $\left\|\left(\nabla \cdot \mathbf{u}_{h}\right)(T)\right\|$ \\
\hline $10^{0}$ & $2.4387-14$ & $3.8077-14$ & $1.2046-14$ & $6.3479-14$ \\
$10^{-2}$ & $2.8404-14$ & $2.9734-14$ & $1.2284-14$ & $5.9303-14$ \\
$10^{-3}$ & 111.6 & $4.2587-14$ & $1.4865-7$ & $5.8514-14$ \\
$10^{-4}$ & 236.5 & 84.8 & $2.0844-7$ & $6.1256-14$ \\
$10^{-5}$ & 318.2 & 159.6 & $2.1986-7$ & $6.0862-14$ \\
$10^{-6}$ & 330.9 & 188.9 & $2.0990-7$ & $1.3439-10$ \\
\hline
\end{tabular}
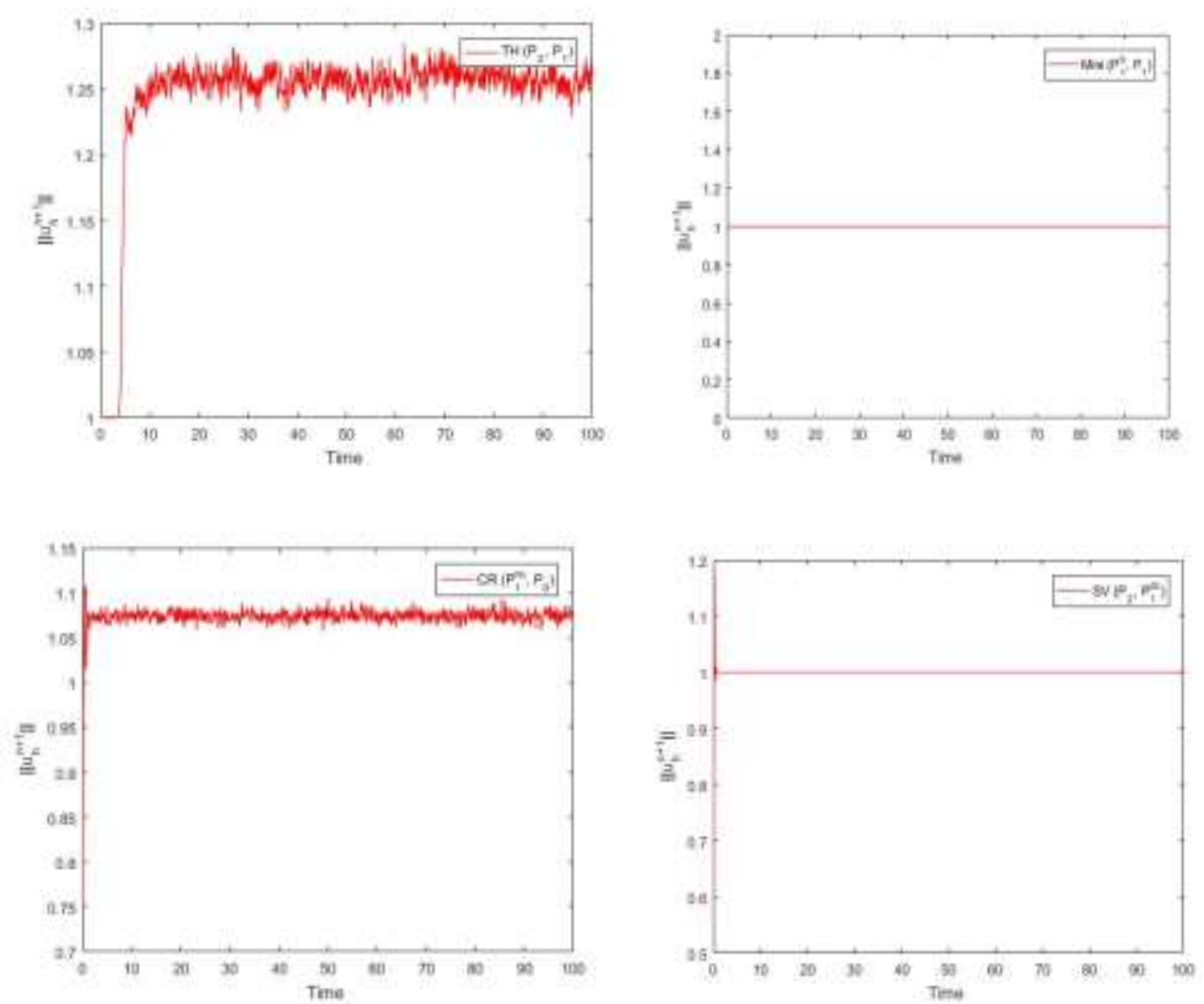

Figure 1. Shown above plots of $L_{2}$-norm of the computed solutions $\left\|u_{h}^{n+1}\right\|_{L^{2}}$ versus time for $v=10^{-3}$. 

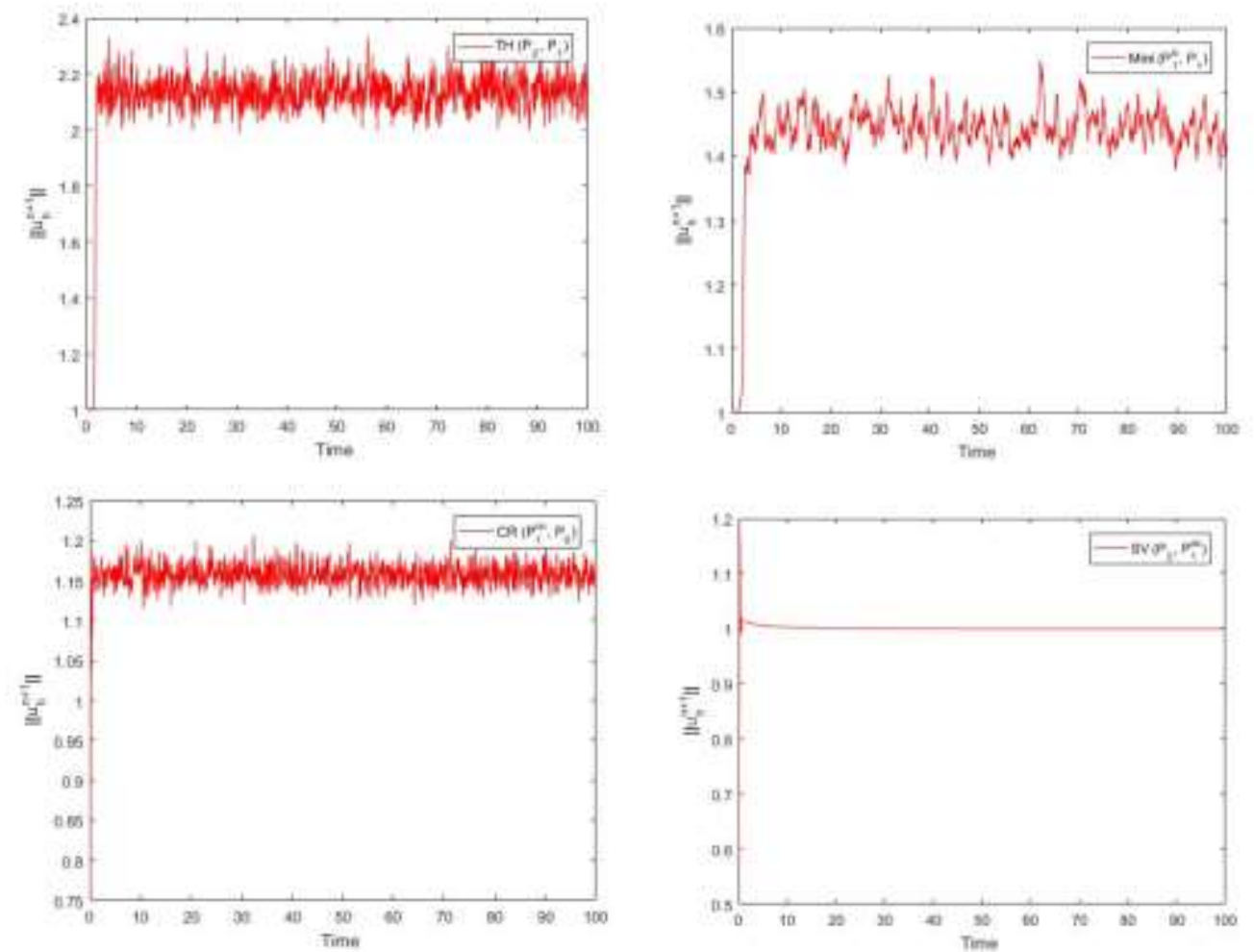

Figure 2. Shown above plots of $\boldsymbol{L}_{2}$-norm of the computed solutions $\left\|\mathbf{u}_{h}^{n+1}\right\|_{L^{2}}$ versus time for $\boldsymbol{v}=\mathbf{1 0}^{\mathbf{- 6}}$.

\subsection{Stability behaviour}

In the second numerical experiment, we show the effect of the filter step. Therefore, we compare the stability of velocity solution of Algorithm 3.1 with the those of standard linearized BE-FEM, i.e.,algorithm without the second step. We use the same flow parameters as in the previous experiment, and run algorithms taking TH-FEM, which is not divergence free, with time step $\mathrm{dt}=0.05$. (We also run $\mathrm{dt}=0.01, \mathrm{dt}=0.25$ and $\mathrm{dt}=0.1$, and got the similar results.) The plots of the computed approximate solutions in the $L_{2}$ - norm are presented in Figure 3 and Figure 4. For $v=1.0$, both algorithms give the exact velocity solution. As $v$ is smaller and smaller, BE-FEM deviates from the exact solution, but THFEM gives the exact velocity solutions. This is due to the fact that filter step increases the accuracy of BE-FEM.
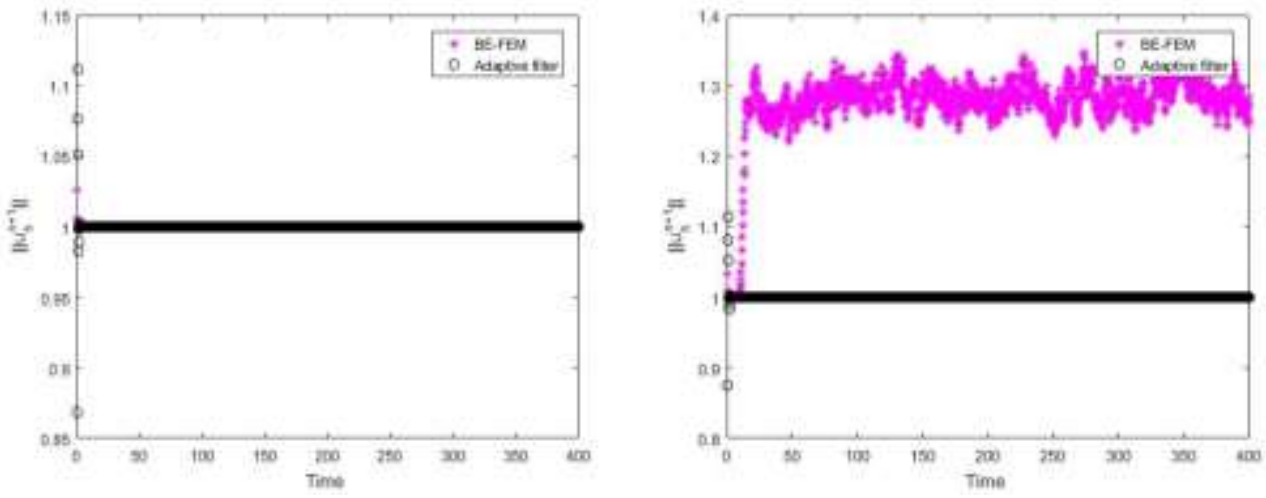

Figure 3. Shown above plots of $L_{2}$-norm of Algorithm 3.1's and BE-FEM's solutions versus time for

$$
v=\frac{1}{100}, \frac{1}{250} .
$$



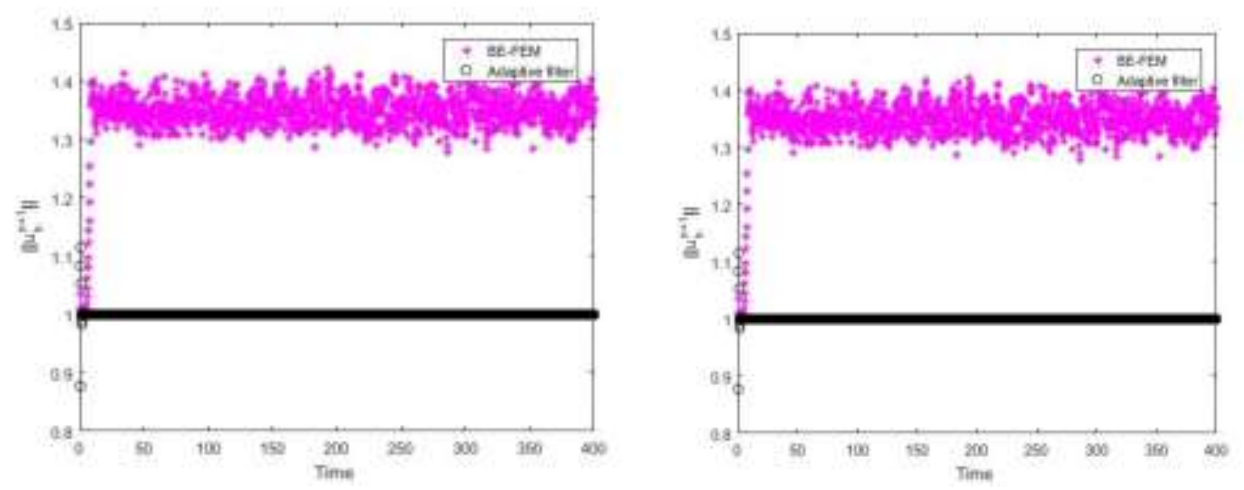

Figure 4. Shown above plots of $\boldsymbol{L}_{\mathbf{2}}$-norm of Algorithm 3.1's and BE-FEM's solutions versus time for

$$
v=\frac{1}{500}, \frac{1}{1000} \text {. }
$$

\section{Yazarların Katkısı}

Çalışmada tüm yazarlar eşit oranda katkı sunmuştur.

\section{Çıkar Çatışması Beyanı}

Yazarlar arasında herhangi bir çıkar çatışması bulunmamaktadır.

\section{Araştırma ve Yayın Etiği Beyanı}

Yapılan çalışmada araştırma ve yayın etiğine uyulmuştur.

\section{Kaynaklar}

[1] Adams R.A. 1975. Sobolev Spaces. Academic Press, New York.

[2] Akbas Belenli M., Rebholz L.G., Tone F. 2015. A Note on the Importance of Mass Conservation in Long-Time Stability of Navier-Stokes Simulations Using Finite Elements. Applied Mathematics Letters, 45: 98-102.

[3] Chen W., Gunzburger M., Sun D., Wang X. 2013. Efficient and Long-Time Accurate SecondOrder Methods for Stokes-Darcy System. SIAM J. Numer. Anal., 51(5): 2563-2584.

[4] Decaria V., Layton W., Zhao H. 2020. A Time Accurate, Adaptive Discretization for Fluid Flow Problems. Inter. J. Numer. Anal. Mod., 17(2): 254-280.

[5] Ewald B., Tone F. 2013. Approximation of the Long-Term Dynamics of the Dynamical System Generated by the Two-Dimensional Thermohydraulies Equations. International Journal of Numerical Analysis and Modelling, 10(3): 509-535.

[6] Girault V., Raviart P.A. 1979. Finite Element Approximation of the Navier-Stokes Equations. Lecture Notes in Mathematics 719, Springer-Verlag, Berlin.

[7] Gottlieb S., Tone F., Wang C., Wang X., Wirosoetisno D. 2012. Long-Time Stability of a Classical Efficient Scheme for Two Dimensional Navier-Stokes Equations. SIAM J. Numer. Anal., 50 (1): 126-150.

[8] Güzel A., Layton W. 2018. Time Filters Increase Accuracy of the Fully Implicit Method. BIT Numer. Math., 58 (2): 301-315.

[9] Heister T., Olshanskii M.A., Rebholz L.G. 2017. Unconditional Long-Time Stability of a Velocity-Vorticity Method for 2D Navier-Stokes Equations. Numer. Math., 135(1): 143-167. 
[10] John V., Linke A., Merdon C., Neilan M., Rebholz L. 2017. On the Divergence Constranint in Mixed Finite Element Methods for Incompressible Flows. SIAM Rev., 59(3): 492-544.

[11] Layton W. 2008. Introduction to Finite Element Methods for Incompressible,Viscous Flow. SIAM, Philadelphia.

[12] Lee H.K., Olshanskii M.A., Rebholz L.G. 2011. On Error Analysis for the 3D Navier-Stokes Equations in Velocity-Vorticity-Helicity Form. SIAM J. Numer. Anal., 49(2): 711-732.

[13] Linke A. 2014. On the role of the Helmholtz Decomposition in Mixed Methods for Incompressible Flows and a New Variational Crime. Comput. Method.Appl. M., 268: 782-800.

[14] Linke A., Merdon C. 2016. Pressure-Robustness and Discrete Helmholtz Projectors in Mixed Finite Element Methods for the Incompressible Navier-Stokes Equations. Comput. Method. Appl.M., 311: 304-326.

[15] Ahmed N., Linke A., Merdon C. 2017. Towards Pressure-Robust Mixed Methods for the Incompressible Navier-Stokes Equations. In Proceedings of the Finite Volumes for Complex Applications 8: 351-359.

[16] Pedlosky J. 1992. Geophysical Fluid Dynamics. Springer, New York.

[17] Tone F. 2007. On the Long-Time Stability of the Crank-Nicolson Scheme for the 2D NavierStokes Equations. Numer. Meth. Part. D.E., 23(5): 1235-1248.

[18] Tone F. 2009. On the Long-Time $H^{2}$-Stability of the Implicit Euler Scheme for the 2D MagnetoHydrodynamics Equations. J. Sci. Comput., 38(3): 331-348.

[19] Tone F., Wirosoetisno D. 2006. On the Long-Time Stability of the Implicit Euler Scheme for the two-dimensional Navier-Stokes Equations, SIAM Journal on Numerical Analysis, 44(1): 29-40.

[20] Wang X. 2012. An Efficient Second Order in Time Scheme for Approximating Long-Time Statistical Properties of the Two Dimensional Navier-Stokes Equations. Numer. Math., 121(4): 753-779. 